\title{
Entanglement versus negative domains of Wigner functions
}

\author{
Dahl, Jens Peder; Mack, H.; Wolf, A.; Schleich, W. P.
}

Published in:

Physical Review A

Link to article, DOI:

10.1103/PhysRevA.74.042323

Publication date:

2006

Document Version

Publisher's PDF, also known as Version of record

Link back to DTU Orbit

Citation (APA):

Dahl, J. P., Mack, H., Wolf, A., \& Schleich, W. P. (2006). Entanglement versus negative domains of Wigner functions. Physical Review A, 74(4), 042323. https://doi.org/10.1103/PhysRevA.74.042323

\section{General rights}

Copyright and moral rights for the publications made accessible in the public portal are retained by the authors and/or other copyright owners and it is a condition of accessing publications that users recognise and abide by the legal requirements associated with these rights.

- Users may download and print one copy of any publication from the public portal for the purpose of private study or research.

- You may not further distribute the material or use it for any profit-making activity or commercial gain

- You may freely distribute the URL identifying the publication in the public portal

If you believe that this document breaches copyright please contact us providing details, and we will remove access to the work immediately and investigate your claim 


\title{
Entanglement versus negative domains of Wigner functions
}

\author{
J. P. Dahl, ${ }^{1,2, *}$ H. Mack, ${ }^{1}$ A. Wolf, ${ }^{1, \dagger}$ and W. P. Schleich ${ }^{1}$ \\ ${ }^{1}$ Abteilung für Quantenphysik, Universität Ulm, D-89069 Ulm, Germany \\ ${ }^{2}$ Chemical Physics, Department of Chemistry, Technical University of Denmark, DTU 207, DK-2800 Kgs. Lyngby, Denmark
}

(Received 18 August 2006; published 23 October 2006)

\begin{abstract}
We show that $s$ waves, that is wave functions that only depend on a hyperradius, are entangled if and only if the corresponding Wigner functions exhibit negative domains. We illustrate this feature using a special class of $s$ waves which allows us to perform the calculations analytically. This class includes a Gaussian, a maximally entangled as well as a "shell" state.
\end{abstract}

DOI: 10.1103/PhysRevA.74.042323

\section{INTRODUCTION}

According to Schrödinger [1] entanglement is the trait of quantum mechanics. The Wigner phase space distribution $[2,3]$ can display negative domains due to quantum correlations between position and momentum. An $s$ wave is built up [4] by interference. In the present paper, we bring these three seemingly distinct facts together and relate entanglement to negative volumes of Wigner functions. Moreover, we study the dependence of entanglement on the number of space dimensions.

Our study concentrates on wave functions which depend on the hyperradius $r$ only. They correspond to angular momentum zero and carry the name $s$ waves. However in order to fully characterize these waves, in particular with regard to their sole dependence on the hyperradius, we call such $s$ waves hyperradial $s$ waves.

The example of two particles which live in $d$ dimensions and whose total angular momentum vanishes bring out most clearly the need for this distinction. Even when both particles have a vanishing angular momentum and are described by wave functions $\phi_{1}$ and $\phi_{2}$ which depend on hyperradius $s_{1}$ and $s_{2}$ in $d$ dimensions the total wave function $\phi_{1}\left(s_{1}\right) \phi_{2}\left(s_{2}\right)$ might not be expressible solely in terms of the hyperradius $r=\sqrt{s_{1}^{2}+s_{2}^{2}}$ in $D=2 d$ dimensions. As a consequence such an $s$ wave is not a hyperradial $s$ wave. The case of a Gaussian wave function is an important exception since the product of two Gaussian $s$ waves involves the hyperradius in $D=2 d$ only.

The Gaussian exception provides also the link to the Wigner function: The only state with a Wigner function which is positive everywhere is a Gaussian. For all other states and in particular all other hyperradial $s$ waves, the Wigner function must take negative values.

Wigner's phase space distribution $[2,3]$ brings out most clearly the interference nature of quantum mechanics. A hyperradial $s$ wave is a wave function, where interference is most prominent [4]. The Wigner function of such an $s$ wave depends on $r$ and the absolute value $k$ of the wave vector, $\vec{k}$, but also on the angle $u$ between $\vec{r}$ and $\vec{k}$. This fact expresses a special correlation between $\vec{r}$ and $\vec{k}$, which manifests itself in negative parts of the corresponding Wigner function.

\footnotetext{
*Electronic address: jpd@kemi.dtu.dk

†Electronic address: alexander.wolf@physik.uni-ulm.de
}

PACS number(s): 03.67.Mn, 03.65.Ud, 42.50.-p

On the other hand, a hyperradial $s$ wave in $D=2 d$ dimensions can be interpreted as a wave function of two entangled particles in $d$ dimensions. Several measures of entanglement [5] offer themselves to quantify the amount of entanglement contained in such an $s$ wave. Here we choose entanglement as determined by the von Neumann entropy [6] and compare and contrast it to the volume [7] contained in the negative parts of the Wigner function. In addition we consider a particular Clauser-Horne-Shimony-Holt (CHSH) sum [8].

On first sight our result seems to be in contradiction with a paper [9] by Bell. He has emphasized that the Wigner function of the prototype of the entangled state, that is, the original Einstein-Podolsky-Rosen (EPR) state [10], is nowhere negative. To be sure, the Wigner function of the EPR state is the product of two delta functions. The problem of the appropriate interpretation of a delta function can be avoided by considering [11] the limiting process of a squeezed two-particle Gaussian. Here indeed the corresponding Wigner function is nowhere negative. However, there is no contradiction to the present work since the EPR wave function is not of the form of an $s$ wave.

The negative domains of Wigner functions as an indicator of the nonclassical nature of a quantum state has recently been proposed. Three approaches to quantify these domains stand out: (i) find bounds on integrals [12] of the Wigner distributions over some subregion or contour of the phase plane for one-dimensional quantum systems, (ii) use the contrast [13], familiar from optics, defined by the moduli of the integrals over the domains of phase space where the Wigner function is positive and negative, and consider the deviation from unity, and (iii) calculate the phase space volume of the difference [7] between the Wigner function and its absolute value. Our measure of negative domains is closely related to the method (iii). However we are not aware of any literature which tries to connect entanglement to the negative domains of the Wigner function.

Recently, papers [14-16] have discussed the question of an appropriate measure of entanglement for identical particles such as bosons and fermions. In contrast to that our analysis focuses on the wave function of the motion and neglects the spin degrees of freedom.

Our paper is organized as follows: In Sec. II we prove that hyperradial $s$ waves are entangled if and only if the corresponding Wigner function assumes negative values. Our proof is based on the Hudson-Piquet theorem [3] generalized [17] to higher dimensions: The only Wigner function that is positive everywhere is a Gaussian. On the other hand the 
only separable hyperradial $s$ state is a Gaussian. We then illustrate our approach using an elementary $s$ wave in $D$ dimensions. For this particular wave function we find in Sec. III the Schmidt decomposition which allows us to evaluate in Sec. IV two measures of the entanglement, namely the von Neumann entropy and the nonclassicality defined by the CHSH sum [8]. We finally turn in Sec. V to the approach based on the Wigner function. For this purpose we first calculate the Wigner function of a hyperradial $s$-wave function. It depends not only on the absolute values of position and momentum but also on the angle between them. The negative domains in phase space clearly depend on this angle. In order to quantify these domains we introduce in Sec. VI the negative volume of the Wigner function and make the connection to the entanglement. We conclude in Sec. VII by summarizing our results and providing an outlook.

\section{A GENERAL ARGUMENT}

In this section we bring to light the intimate connection between the negative domains of the Wigner function and entanglement, which exists for hyperradial $s$ waves. For this purpose we consider two particles each of which live in $d$ dimensions, that is, $D \equiv 2 d$ and $\vec{r}=\left(\vec{s}_{1}, \vec{s}_{2}\right)$ leading to the $s$-wave function

$$
\Psi^{(D)}(\vec{r}) \equiv \Psi^{(D)}(r) \equiv \Psi^{(2 d)}\left(\vec{s}_{1}, \vec{s}_{2}\right) \equiv \Psi^{(2 d)}\left(\sqrt{s_{1}^{2}+s_{2}^{2}}\right) .
$$

We define a hyperradial $s$ wave which is separable by the condition that the wave function

$$
\Psi\left(s_{1}, s_{2}\right)=\phi_{1}\left(s_{1}\right) \phi_{2}\left(s_{2}\right)
$$

factorizes into a product of two wave functions corresponding to two separable particles. Obviously a Gaussian satisfies this functional relationship. However, we now recall [18] that a Gaussian is the only function that satisfies this condition.

Since $s_{1}$ and $s_{2}$ enter the wave function $\Psi^{(D)}$ in a symmetric way through $r \equiv\left(s_{1}^{2}+s_{2}^{2}\right)^{1 / 2}$ we find with the help of the chain rule

$$
\frac{1}{s_{1}} \frac{\partial \Psi(r)}{\partial s_{1}}=\frac{\partial \Psi(r)}{\partial r} \frac{1}{r}=\frac{1}{s_{2}} \frac{\partial \Psi(r)}{\partial r} \frac{\partial r}{\partial s_{2}} .
$$

The product representation, Eq. (2), translates this relation into

$$
\frac{1}{s_{1}} \frac{\partial \phi_{1}\left(s_{1}\right)}{\partial s_{1}} \phi_{2}\left(s_{2}\right)=\frac{1}{s_{2}} \frac{\partial \phi_{2}\left(s_{2}\right)}{\partial s_{2}} \phi_{1}\left(s_{1}\right)
$$

which after division by $\phi_{1}\left(s_{1}\right) \phi_{2}\left(s_{2}\right)$ yields the condition

$$
\frac{1}{s_{1}} \frac{\partial \phi_{1}\left(s_{1}\right)}{\partial s_{1}} \frac{1}{\phi_{1}\left(s_{1}\right)}=\frac{1}{s_{2}} \frac{\partial \phi_{2}\left(s_{2}\right)}{\partial s_{2}} \frac{1}{\phi_{2}\left(s_{2}\right)} \equiv-\alpha .
$$

In the last step we have used the familiar argument in separating variables and have introduced the constant $\alpha$ of separation.

The unique normalizable solution of Eq. (5) reads

$$
\phi_{1}(s)=\phi_{2}(s)=\mathcal{N} e^{-\alpha s^{2} / 2},
$$

where $\mathcal{N}$ is a normalization constant. Consequently the Gaussian state is the only hyperradial $s$ state that is separable.

On the other hand, Gaussian states of the form

$$
\Psi(\vec{r})=\mathcal{N} \exp (-\vec{r} \cdot \mathbf{A} \cdot \vec{r}+\vec{b} \cdot \vec{r})
$$

are the only states whose Wigner functions are positive everywhere [17]. Here $\mathbf{A}$ is a self-adjoint positive definite matrix and $\vec{b}$ is a complex $D$-dimensional vector. Only in the case that $\mathbf{A}$ is proportional to the unit matrix and $\vec{b}=\overrightarrow{0}$ is the state $\Psi(\vec{r})$ an $s$ state.

Hence, the only hyperradial $s$ state that is separable has a Wigner function which is non-negative. Therefore, hyperradial $s$ states are entangled if and only if the corresponding Wigner function assumes negative domains.

\section{ELEMENTARY $s$ WAVE}

Our analysis of entanglement contained in $s$ waves is based on an elementary $s$-wave function introduced in the present section. We first briefly summarize important properties of this wave function and then derive the eigenvalues of the one-particle reduced density matrix necessary for calculating the entanglement discussed in the next section.

\section{A. Definition}

The wave function in $D$ dimensions that forms the basis of the present discussion reads

$$
\Psi^{(D)}(\vec{r}) \equiv \Psi^{(D)}(r) \equiv \mathcal{N}_{a}^{(D)}\left(1+a r^{2}\right) \pi^{-D / 4} e^{-(1 / 2) r^{2}},
$$

where

$$
\mathcal{N}_{a}^{(D)} \equiv\left[\left(1+\frac{1}{2} a D\right)^{2}+\frac{1}{2} a^{2} D\right]^{-1 / 2}
$$

and $r \equiv \sqrt{x_{1}^{2}+x_{2}^{2}+\cdots+x_{D}^{2}}$.

This function reduces to the Gaussian

$$
\Psi_{G}^{(D)}(r)=\pi^{-D / 4} e^{-(1 / 2) r^{2}}
$$

for $a=0$ and to the "shell" wave function

$$
\Psi_{S}^{(D)}(r)=\mathcal{N}_{S}^{(D)} \pi^{-D / 4} r^{2} e^{-(1 / 2) r^{2}}
$$

when we take the limit $a \rightarrow \pm \infty$ with

$$
\mathcal{N}_{S}^{(D)} \equiv \lim _{a \rightarrow \infty} a \mathcal{N}_{a}^{(D)}=2[D(D+2)]^{-1 / 2} .
$$

Hence, the parameter $a$ interpolates between the two extremes, that is the Gaussian and the "shell" wave function.

The two functions $\Psi_{G}^{(D)}$ and $\Psi_{S}^{(D)}$ have also been studied in other contexts. In particular, emphasis has been put on the dependence of their kinetic energies [19] and their time evolution [20] in the absence of external fields, on the number $D$ of dimensions.

The wave function 


$$
\Psi_{\max }^{(D)}(r)=\mathcal{N}_{\max }^{(D)}\left(1-\frac{2}{D} r^{2}\right) \pi^{-D / 4} e^{-(1 / 2) r^{2}}
$$

with $\mathcal{N}_{\max }^{(D)} \equiv(D / 2)^{1 / 2}$ which emerges from the elementary $s$ wave, Eq. (8), for $a=-2 / D$ will play an important role throughout the paper. The corresponding state is the orthogonal complement to the Gaussian in the space spanned by the functions in Eq. (8). In the next section we will show that $\Psi_{\max }^{(D)}$ corresponds to a maximally entangled state.

Instead of the function $\Psi^{(D)}$ defined by Eq. (8), we might consider the scaled wave function

$$
\Psi^{(D)}(r) \equiv \mathcal{N}_{a}^{(D)}\left[1+a(\kappa r)^{2}\right]\left(\kappa^{2} / \pi\right)^{D / 4} e^{-(1 / 2)(\kappa r)^{2}},
$$

where $\kappa$ is real and positive. It is, however, easy to see that all three measures that we consider in this work, namely, the entanglement, the $\mathrm{CHSH}$ sum, and the negative part of Wigner function, are independent of $\kappa$. Hence, we consider only the function of Eq. (8), corresponding to $\kappa=1$.

\section{B. State vector representation}

In the first step of our analysis we use a complete set of orthonormal functions in $d \equiv D / 2$ dimensions to express $\Psi^{(D)}$ as an entangled two-particle state vector. In this way we map the quantum system with continuous variables onto one with discrete variables.

For $\vec{r}=\left(\vec{s}_{1}, \vec{s}_{2}\right)$ the elementary $s$ wave, Eq. (8), reads

$$
\Psi^{(2 d)}\left(s_{1}, s_{2}\right)=\mathcal{N}_{a}^{(2 d)}\left(1+a s_{1}^{2}+a s_{2}^{2}\right) \pi^{-d / 2} e^{-(1 / 2)\left(s_{1}^{2}+s_{2}^{2}\right)} .
$$

The orthonormal set of functions

$$
\varphi_{0}(s)=\pi^{-d / 4} e^{-(1 / 2) s^{2}}
$$

and

$$
\varphi_{2}(s)=\left(\frac{d}{2}\right)^{1 / 2}\left(1-\frac{2}{d} s^{2}\right) \pi^{-d / 4} e^{-(1 / 2) s^{2}}
$$

allows us to expand $\Psi^{(2 d)}\left(s_{1}, s_{2}\right)$ as the sum

$$
\begin{aligned}
\Psi^{(2 d)}\left(s_{1}, s_{2}\right)= & c_{00} \varphi_{0}\left(s_{1}\right) \varphi_{0}\left(s_{2}\right)+c_{02} \varphi_{0}\left(s_{1}\right) \varphi_{2}\left(s_{2}\right) \\
& +c_{20} \varphi_{2}\left(s_{1}\right) \varphi_{0}\left(s_{2}\right)
\end{aligned}
$$

with the coefficients

$$
c_{00} \equiv \mathcal{N}_{a}^{(2 d)}(1+a d)=\frac{1+a d}{\left[(1+a d)^{2}+a^{2} d\right]^{1 / 2}}
$$

and

$$
c_{20}=c_{02} \equiv-\mathcal{N}_{a}^{(2 d)} \frac{a \sqrt{d}}{\sqrt{2}}=-\frac{1}{\sqrt{2}} \frac{a \sqrt{d}}{\left[(1+a d)^{2}+a^{2} d\right]^{1 / 2}} .
$$

The abbreviations

$$
|0\rangle \equiv \varphi_{0} \quad \text { and } \quad|2\rangle \equiv \varphi_{2} \quad \text { and } \quad|\Psi\rangle \equiv \Psi^{(D)}
$$

bring to light that the $s$ wave, Eq. (8), corresponds to the two-qubit entangled state

$$
|\Psi\rangle=c_{00}|00\rangle+c_{20}|20\rangle+c_{02}|02\rangle .
$$

We emphasize that for $c_{00}=0$, which is equivalent to the condition

$$
a=-\frac{1}{d}
$$

on the interpolation parameter $a$, the state $|\Psi\rangle$ reduces to a maximally entangled Bell state

$$
|\Psi\rangle_{\max }=\frac{1}{\sqrt{2}}(|02\rangle+|20\rangle) .
$$

\section{Schmidt decomposition}

In the next step of our analysis we calculate the eigenvalues $\lambda_{i}$ of the one-particle reduced density operator

$$
\begin{aligned}
\hat{\rho}_{1} & =\operatorname{Tr}_{2}\{|\Psi\rangle\langle\Psi|\} \\
& =\left(c_{00}^{2}+c_{02}^{2}\right)|0\rangle\left\langle 0\left|+c_{20} c_{00}\right| 2\right\rangle\left\langle 0\left|+c_{00} c_{20}\right| 0\right\rangle\left\langle 2\left|+c_{20}^{2}\right| 2\right\rangle\langle 2|
\end{aligned}
$$

following from the eigenvalue equation

$$
\hat{\rho}_{1}\left|\psi_{j}\right\rangle=\lambda_{j}\left|\psi_{j}\right\rangle
$$

with the eigenfunctions $\left|\psi_{j}\right\rangle$. The reduced density matrix for the first particle reads

$$
\rho_{1}=\left(\begin{array}{cc}
c_{00}^{2}+c_{20}^{2} & c_{20} c_{00} \\
c_{20} c_{00} & c_{20}^{2}
\end{array}\right)
$$

and yields the eigenvalue equation

$$
\lambda^{2}-\lambda+c_{02}^{4}=0
$$

where we have made use of the definitions, Eqs. (19) and (20), of $c_{00}$ and $c_{20}$, respectively, to show the identity

$$
c_{00}^{2}+2 c_{20}^{2}=1
$$

Hence, the eigenvalues take the form

$$
\lambda_{ \pm}=\frac{1}{2}\left\{1 \pm\left[1-\left(\frac{a^{2} d}{(1+a d)^{2}+a^{2} d}\right)^{2}\right]^{1 / 2}\right\} .
$$

We are now in the position to obtain the Schmidt decomposition [21]

$$
|\Psi\rangle=\sum_{j= \pm} \sqrt{\lambda_{j}}\left|\psi_{j}\right\rangle\left|\varphi_{j}\right\rangle
$$

of the $s$-wave state $|\Psi\rangle$. Here $\left|\psi_{j}\right\rangle$ and $\left|\varphi_{j}\right\rangle$ are the normalized eigenvectors of the reduced density matrices for particles 1 and 2, respectively. Since the elementary $s$ wave, Eq. (15), is symmetric in the two particles, the members of the two sets of eigenvectors may only differ by a phase factor, that is, $\left|\psi_{j}\right\rangle=e^{i \delta_{j}}\left|\varphi_{j}\right\rangle$. Moreover $|\Psi\rangle$ is real. Hence, only the phases $\delta_{j}=0$ and $\delta_{j}=\pi$ are possible.

\section{MEASURES OF ENTANGLEMENT}

We start our discussion of the relation between entanglement and the negative domains of the Wigner function by 
first calculating for the $s$ wave, Eq. (8), the entanglement as defined by the von Neumann entropy $E\left(\Psi^{(D)}\right)$. Moreover we use the Schmidt decomposition, Eq. (31), to calculate the maximal value of the Clauser-Horne-Shimony-Holt (CHSH) sum $S\left(\Psi^{(D)}\right)$ as a measure of nonclassicality. Both $E\left(\Psi^{(D)}\right)$ and $S\left(\Psi^{(D)}\right)$ depend on the number $D$ of dimensions and the interpolation parameter $a$ in a very similar way.

\section{A. von Neumann entropy}

As a measure of the entanglement $E$ of pure states we use the von Neumann entropy [6]

$$
E \equiv-\operatorname{Tr}\left(\hat{\rho}_{1} \log _{2} \hat{\rho}_{1}\right)=-\sum_{j} \lambda_{j} \log _{2} \lambda_{j} .
$$

Since for the $s$ wave, Eq. (8), the one-particle reduced density matrix, Eq. (27), has just the two eigenvalues $\lambda_{ \pm}$given by Eq. (30) the entanglement simplifies to

$$
E\left(\Psi^{(D)}\right)=-\lambda_{+} \log _{2} \lambda_{+}-\lambda_{-} \log _{2} \lambda_{-} .
$$

On the top of Fig. 1 we show the dependence of the von Neumann entropy $E\left(\Psi^{(D)}\right)$ on the interpolation parameter $a$ and the dimension $D=2 d$. We recognize that the entanglement vanishes for $a=0$, that is for the Gaussian state, and reaches its maximum at $a=-2 / D=-1 / d$. It is interesting to note that for this simple example of an $s$ state the maximally entangled state can be reached for any dimension $D$.

\section{B. Nonclassicality}

The Schmidt decomposition, Eq. (31), is a discrete representation of the $s$ wave, Eq. (8). We may compare this form with the representation

$$
|\Psi\rangle=\alpha|\uparrow\rangle|\uparrow\rangle+\beta|\downarrow\rangle|\downarrow\rangle
$$

of an entangled state of two spin- $-\frac{1}{2}$ particles. Here $|\uparrow\rangle$ and $|\downarrow\rangle$ represent spins polarized along axes which may differ from one particle to the other and $\alpha$ and $\beta$ may be chosen to be real with $\alpha^{2}+\beta^{2}=1$.

It has been shown [22-24] that for such a state the Clauser-Horne-Shimony-Holt (CHSH) sum [8], $S(\Psi)$, of four particular expectation values has the maximum value

$$
S(\Psi)=2 \sqrt{1+4 \alpha^{2} \beta^{2}} .
$$

The extent to which this value exceeds 2 is a measure of nonclassical correlations. For all nonvanishing values of $\alpha$ and $\beta, S(\Psi)$ lies between 2 and $2 \sqrt{2}$. The value 2 is obtained when either $\alpha$ or $\beta$ is equal to zero corresponding to no entanglement. The value $2 \sqrt{2}$ is obtained when $\alpha=\beta=1 / \sqrt{2}$ and corresponds to maximum entanglement.

When we compare the Schmidt decomposition Eq. (31) with the entangled state Eq. (34) we can identify $\alpha^{2}=\lambda_{+}$and $\beta^{2}=\lambda_{-}$which yields

$$
S\left(\Psi^{(D)}\right)=2\left[1+\left(\frac{a^{2} d}{(1+a d)^{2}+a^{2} d}\right)^{2}\right]^{1 / 2} .
$$

In the middle part of Fig. 1 we show the nonclassicality of the $s$ wave, Eq. (8), expressed by $S\left(\Psi^{(D)}\right)$ as a function of
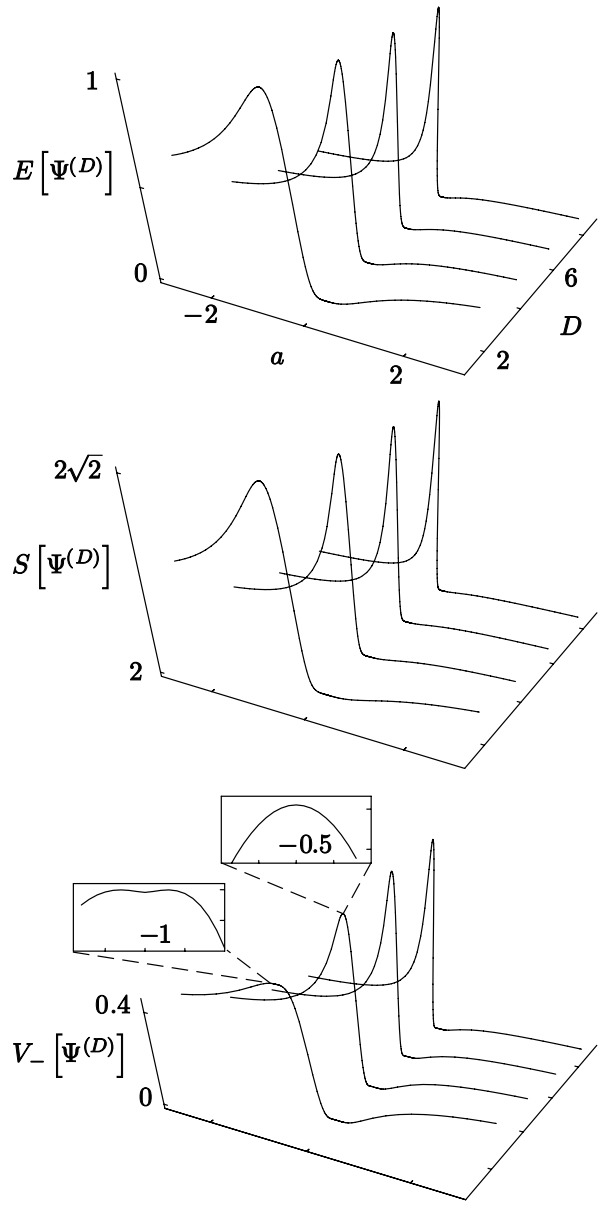

FIG. 1. Comparison between three different measures of the entanglement contained in the elementary $s$ wave, Eq. (8), as a function of the interpolation parameter $a$ and number $D=2 d$ of dimensions: The von Neumann entropy, Eq. (33) (top), the maximal value of the CHSH sum $S\left(\Psi^{(D)}\right)$, Eq. (36) (middle), and the numerically calculated negative volume $V_{-}\left(\Psi^{(D)}\right)$, Eq. (47) (bottom), of the Wigner function corresponding to the elementary $s$ wave. All three figures are similar in their characteristic features. In particular, all three measures vanish for a Gaussian, that is $a=0$, and reach their maximal values $E=1, S=2 \sqrt{2}$ at the critical point $a=-2 / D$. The only exception to this behavior appears in two dimensions, where the negative volume $V_{-}\left(\Psi^{(D)}\right)$ has a local minimum at $a=-1$ as shown on the left-hand inset. In all other dimensions we observe an absolute maximum of $V_{-}\left(\Psi^{(D)}\right)$ corresponding to maximally entangled states as shown by the example of $D=4$ in the neighborhood of $a=-0.5$. On the left-hand inset the separation of the ticks on the vertical and horizontal axes is $1 \times 10^{-3}$ and $5 \times 10^{-2}$, respectively. On the right-hand inset the separation of the ticks on the vertical and horizontal axes is $3 \times 10^{-3}$ and $5 \times 10^{-3}$, respectively. The horizontal axes on three figures are identical.

dimension $D=2 d$ and the interpolation parameter $a$. In complete agreement with the entanglement $E\left(\Psi^{(D)}\right), S\left(\Psi^{(D)}\right)$ vanishes for a Gaussian, that is $a=0$, and reaches its maximum value $S\left(\Psi^{(D)}\right)=2 \sqrt{2}$ for $a=-2 / D=-1 / d$.

We conclude by noting that the agreement between the two measures of entanglement, that is the von Neumann entropy and the maximal value of the CHSH sum, in the case 
of a pure qubit state such as the one given by Eq. (34) has already been shown in Ref. [22].

\section{WIGNER FUNCTION OF $s$ WAVES}

We now turn to the discussion of the relation between entanglement and the negative parts of the Wigner function. For this purpose we first briefly discuss the Wigner function of the elementary $s$ wave, Eq. (8), and focus on the domains of phase space where the Wigner function takes on negative values.

\section{A. General expression}

We recall [2] the definition

$$
W^{(D)} \equiv \frac{1}{(2 \pi)^{D}} \int d^{D} \xi \Psi^{*}\left(\vec{r}-\frac{1}{2} \vec{\xi}\right) \Psi\left(\vec{r}+\frac{1}{2} \vec{\xi}\right) e^{-i \vec{k} \cdot \vec{\xi}}
$$

of the Wigner function $W^{(D)} \equiv W^{(D)}(\vec{r}, \vec{k})$.

For the elementary $s$ wave, Eq. (8), of our study we can perform the integrations analytically using hyperspherical coordinates $[25,26]$ and known Fourier transforms and find [27]

$$
W_{a}^{(D)}=\pi^{-D} e^{-r^{2}-k^{2}}\left(1+a \mathcal{P}_{a}^{(D)}\right),
$$

where the polynomial

$$
\mathcal{P}_{a}^{(D)} \equiv \wp_{-} r^{2}-\wp_{+} k^{2}+\wp\left(r^{2}+k^{2}\right)^{2}-4 \wp k^{2} r^{2} \sin ^{2} u
$$

contains the coefficients

$$
\wp_{ \pm}(a, D) \equiv 2 \frac{1+a D / 2 \pm a}{(1+a D / 2)^{2}+a^{2} D / 2}
$$

and

$$
\wp(a, D) \equiv \frac{a}{(1+a D / 2)^{2}+a^{2} D / 2} .
$$

Hence, the Wigner function depends on $r \equiv|\vec{r}|, k \equiv|\vec{k}|$ and on the angle $u$ between $\vec{r}$ and $\vec{k}$, that is, $W^{(D)}(\vec{r}, \vec{k})$ $=W^{(D)}(r, k, u)$. Moreover, $W^{(D)}$ contains an explicit dependence on the interpolation parameter $a$ and on the number $D$ of dimensions.

\section{B. Special examples}

In order to study this dependence we now consider three limiting cases of $a$.

\section{Separable Gaussian}

We start with the case of a Gaussian, that is $a=0$, which reduces Eq. (38) to the Gaussian Wigner function

$$
W_{a=0}^{(D)}=\pi^{-D} e^{-r^{2}-k^{2}}
$$

which is positive everywhere.

\section{2. "Shell" wave function}

Next we consider the limit $a \rightarrow \pm \infty$ of Eq. (38) leading to the Wigner function

$$
W_{\infty}^{(D)} \equiv W_{S}^{(D)}=\pi^{-D} e^{-r^{2}-k^{2}}\left(1+\frac{4}{D} \mathcal{P}_{S}^{(D)}\right)
$$

of the "shell" function Eq. (11). The polynomial

$$
\begin{aligned}
\mathcal{P}_{S}^{(D)} \equiv & \frac{2 D}{D+2} r^{2}+\frac{1}{D+2}\left(r^{2}+k^{2}\right)^{2}-\left(r^{2}+k^{2}\right) \\
& -\frac{4}{D+2} k^{2} r^{2} \sin ^{2} u
\end{aligned}
$$

contains terms which create domains in phase space where $W_{S}^{(D)}$ is negative. Indeed, the last two contributions to $\mathcal{P}_{S}^{(D)}$ in Eq. (44) are negative. However, for increasing number $D$ of space dimensions the influence of the negative regions decreases due to the prefactor of $\mathcal{P}_{S}^{(D)}$ in Eq. (43) as well as due to the inverse dependence of the prefactor of the last term in $\mathcal{P}_{S}^{(D)}$. On the other hand, according to Eq. (44) the prefactor of the first positive term to $\mathcal{P}_{S}^{(D)}$ is smallest for $D=2$ where it is equal to 1 and approaches 2 for $D \rightarrow \infty$. Thus we suspect that the influence of the negative domains in phase space is most pronounced for $D=2$.

Moreover, the angle $u$ plays a crucial role in the emergence of negative domains. It enters into the Wigner function through the last term of $\mathcal{P}_{S}^{(D)}$ in Eq. (44) in the form of the square of a sine function. Therefore, this contribution vanishes for motions away from and through the origin, that is for $u=0$ and $u=\pi$. In the neighborhood of these angles the negative parts of the Wigner function are not as pronounced as they are for $u=\pi / 2$ corresponding to circular motion.

On the top left-hand side of Fig. 2 we show the Wigner function $W_{S}^{(D)}(r, k, u)$ multiplied by the appropriate factor $r^{D-1} k^{D-1}(\sin u)^{D-2} S^{(D)} S^{(D-1)}$ from the volume element in $D$ dimensions. Here $S^{(D)} \equiv 2 \pi^{D / 2} / \Gamma(D / 2)$ denotes [25] the surface of a unit sphere in $D$ dimensions.

To be specific we have chosen the dimension $D=4$ and the specific angle $u=5 \pi / 12$. We note a dominant positive maximum and a characteristic negative domain. The fat contour line on the bottom of the figure marks the border between positive and negative domains. This border depends sensitively on the angle $u$ between position and momentum.

In order to bring this feature out most clearly we show on the top right-hand side of Fig. 2 the contour lines of the Wigner function separating positive from negative domains for seven angles $u$ starting from $u=0$ to $u=\pi / 2$ in steps of $\pi / 12$. We note that for increasing $u$ the closed domain of negativity increases until at $u=\pi / 2$ it opens up to a canal.

\section{Maximally entangled state}

Finally we turn to the maximally entangled state emerging from the elementary $s$ wave, Eq. (8), for $a=-2 / D$. Here the Wigner function, Eq. (38), takes the form

$$
W_{\max }^{(D)}=\pi^{-D} e^{-r^{2}-k^{2}}\left(1+\frac{4}{D} \mathcal{P}_{\max }^{(0)}\right)
$$

with 

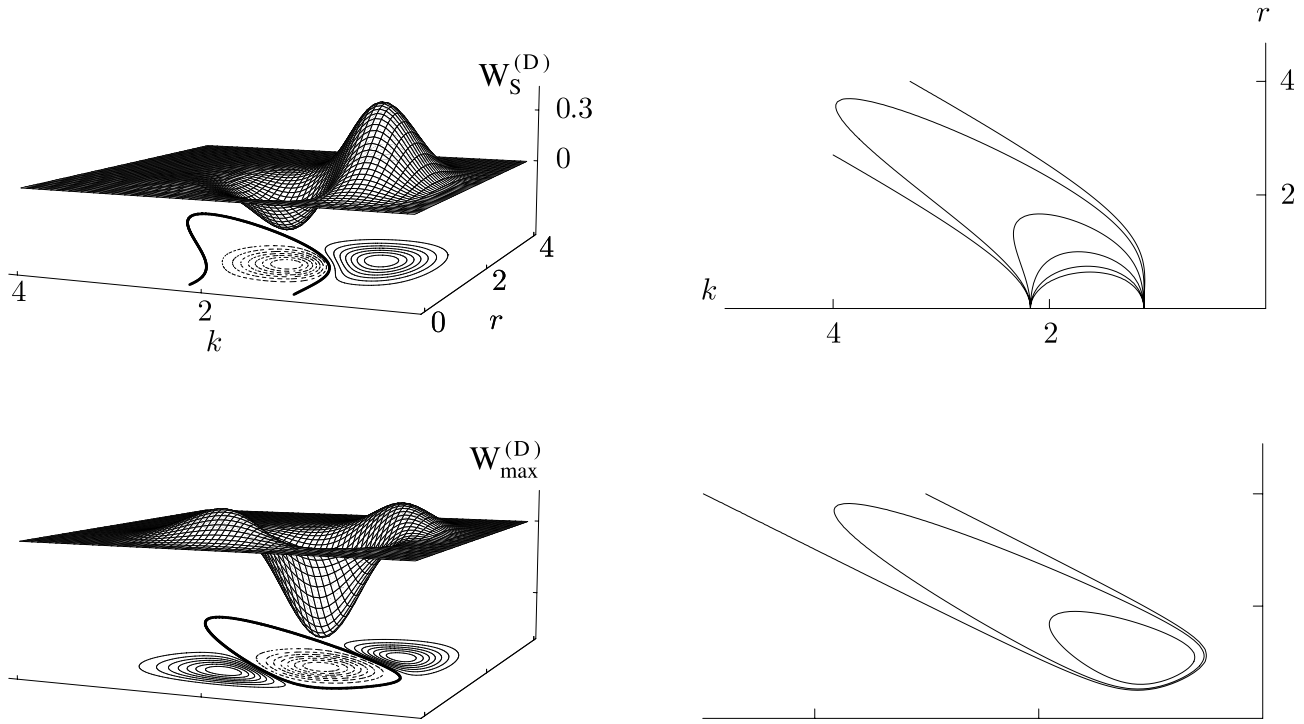

FIG. 2. Wigner functions $W_{S}^{(D)}$ (top left) and $W_{\max }^{(D)}$ (bottom left) given by Eqs. (43) and (45) and corresponding to the "shell" wave function Eq. (11) and the maximally entangled state Eq. (13), respectively, multiplied by the appropriate factor $r^{D-1} k^{D-1}(\sin u)^{D-2} S^{(D)} S^{(D-1)}$ representing the volume element in $D$ dimensions. Both distributions display dominant positive maxima and a characteristic negative domain. The latter is clearly more pronounced at the expense of the positive contributions in the case of the Wigner function of the maximally entangled state. Contour lines on the bottom emphasize the positive regions (solid thin curves) and the negative regions (dotted curves) as well as the border line (fat curve) between them. For both figures in the left-hand column we have chosen the dimension $D=4$ and the angle $u$ between $\vec{r}$ and $\vec{k}$ equal to $5 \pi / 12$. Border lines separating positive regions of $W_{S}^{(D)}$ (top right) and $W_{\max }^{(D)}$ (bottom right) from negative ones for different angles $u$ but fixed dimension $D=4$. In the case of $W_{S}^{(D)}$ all border lines connect the same points on the $k$ axis. As the angle $u$ increases starting from $u=0$ in steps of $\pi / 12$ the border lines circumnavigate increasing volumes in phase space. When $u=0$ the negative domain of the Wigner function is smallest. In the other extreme, that is, for $u=\pi / 2$ we find an open canal. For angles $u$ smaller than $\pi / 4$ the Wigner function $W_{\max }^{(D)}$ of the maximally entangled state is always positive.

$$
\mathcal{P}_{\max }^{(0)} \equiv \frac{1}{2}\left(r^{2}+k^{2}\right)^{2}-\left(r^{2}+k^{2}\right)-2 k^{2} r^{2} \sin ^{2} u .
$$

When we compare this Wigner function to the one of the "shell" wave we find a rather close analogy. The expressions for $W_{S}^{(D)}$ and $W_{\max }^{(D)}$ given by Eqs. (43) and (45) are identical. The only difference between these formulas lies in the polynomials $\mathcal{P}_{S}^{(D)}$ and $\mathcal{P}_{\max }^{(0)}$ defined by Eqs. (44) and (46), respectively. It is remarkable that $\mathcal{P}_{\max }^{(0)}$ is independent of the number $D$ of dimensions. Even more remarkable is the fact that we obtain $\mathcal{P}_{\max }^{(0)}$ from $\mathcal{P}_{S}^{(D)}$ by setting $D=0$. Indeed, in this case the first positive term in $\mathcal{P}_{S}^{(D)}$ disappears and the prefactor $4 /(D+2)$ of the negative contribution due to the angle $u$ assumes its maximal value 2 .

These features suggest that the domains of phase space where the Wigner function becomes negative should be larger for $W_{\max }^{(D)}$ than for $W_{S}^{(D)}$. The bottom left-hand side of Fig. 2 shows the Wigner function $W_{\max }^{(D)}$ for $D=4$ and the angle $u=5 \pi / 12$ and confirms this suggestion. In comparison to the Wigner function $W_{S}^{(D)}$ of the "shell" wave displayed on the top left-hand side of Fig. 2 the negative parts are much more pronounced.

However, from the definition Eq. (44) of $\mathcal{P}_{S}^{(D)}$ we also recognize that the second positive term $\left(r^{2}+k^{2}\right)^{2}$ is most effective when its prefactor $(D+2)^{-1}$ is largest, that is, for $D=0$. Indeed this behavior emerges on the bottom right-hand side of Fig. 2 which depicts the border lines separating positive from negative domains of $W_{\max }^{(D)}$ in their dependence on the angle $u$ between $\vec{r}$ and $\vec{k}$. In sharp contrast to the corresponding picture for $W_{S}^{(D)}$ shown on the top $W_{\max }^{(D)}$ is positive in the center of $r-k$ plane for angles $u<\pi / 4$. Only for the range $\pi / 4<u<3 \pi / 4$ of the angle $u$ the Wigner function $W_{\max }^{(D)}$ takes negative values, however now reaching much larger negative values.

\section{ENTANGLEMENT FROM NEGATIVE DOMAINS OF WIGNER FUNCTIONS}

In Sec. II we have brought to light the intimate connection between the negativity of the Wigner function and the entanglement appearing for hyperradial $s$ waves. We now investigate this relation in a more quantitative way.

For this purpose we evaluate numerically the negative volume $[7,20]$

$$
V_{-} \equiv \frac{1}{2} \int d^{D} x \int d^{D} k|W(\vec{x}, \vec{k})|-\frac{1}{2}
$$

of the Wigner function, Eq. (38).

In the bottom part of Fig. 1 we show the dependence of $V_{-}\left(\Psi^{(D)}\right)$ on the number $D$ of dimensions and the interpolation parameter $a$. In accordance with the entanglement $E\left(\Psi^{(D)}\right)$ and CHSH sum $S\left(\Psi^{(D)}\right)$ we find that for a Gaussian, that is for $a=0$, the negative volume $V_{-}\left(\Psi^{(D)}\right)$ vanishes in any dimensions. Furthermore for $2<D$ the negative volume $V_{-}\left(\Psi^{(D)}\right)$ decreases and increases with decreasing and in- 
creasing entanglement. This behavior also appears for $D=2$, except that there is a minute dip in the negative volume at the critical point $a=-2 / D$ shown on the left-hand inset of Fig. 1.

\section{CONCLUSIONS}

For the case of hyperradial $s$ waves there exists an intimate connection between the two most prominent signatures of the quantum world, that is entanglement and negativity of the Wigner functions. Consequently, the Wigner function of an $s$ state has negative domains if, and only if, this state is entangled. Furthermore, with the help of the elementary $s$ wave, Eq. (8), we have demonstrated that the correspondence between entanglement and the negativity of the Wigner function is not only qualitative but quantitative. It turns out that the negative volume, which we have used as a measurement of the negativity, obeys the same dependence on the number of dimensions and on the interpolation parameter of the elementary $s$ wave, as entanglement. The only exception to this rule appears in the case of two-dimensional quantum systems. This peculiarity of the two-dimensional systems is reminiscent of the anticentrifugal force which is attractive for $D=2$ but repulsive for $D>2$ [20].

\section{ACKNOWLEDGMENTS}

The authors thank J. I. Cirac, M. Freyberger, I. Jex, and S. Varro for many fruitful discussions and K. Życzkowski for providing a copy of Ref. [7]. Moreover, the authors appreciate the support of Landesstiftung Baden-Württemberg in the framework of the Quantum Information Highway A8. One of the authors (A.W.) is thankful for a stipend according to the Landesgraduiertenförderungsgesetz. The authors are also grateful to the Alexander von Humboldt-Stiftung. Moreover, this work was also supported in part by the Max Planck Society.
[1] E. Schrödinger, Proc. Cambridge Philos. Soc. 31, 555 (1935).

[2] E. P. Wigner, Phys. Rev. 40, 749 (1932).

[3] See, for example, W. P. Schleich, Quantum Optics in Phase Space (Wiley-VCH, Weinheim, 2001).

[4] J. P. Dahl, D. M. Greenberger, M. J. Hall, G. Süßmann, A. Wolf, and W. P. Schleich, Laser Phys. 15, 18 (2005).

[5] See, for example, S. Stenholm and K.-A. Suominen, Quantum Approach to Informatics (Wiley, New York, 2005).

[6] S. Parker, S. Bose, and M. B. Plenio, Phys. Rev. A 61, 032305 (2000).

[7] A. Kenfack and K. Życzkowski, J. Opt. B: Quantum Semiclassical Opt. 6, 396 (2004).

[8] J. F. Clauser, M. A. Horne, A. Shimony, and R. A. Holt, Phys. Rev. Lett. 23, 880 (1969).

[9] J. S. Bell, Speakable and Unspeakable in Quantum Mechanics (Cambridge University Press, Cambridge, 1988), Chap. 21, p. 196.

[10] A. Einstein, B. Podolsky, and N. Rosen, Phys. Rev. 47, 777 (1935).

[11] B. G. Englert and K. Wódkiewicz, Phys. Rev. A 65, 054303 (2002).

[12] A. J. Bracken, H.-D. Döbner, and J. G. Wood, Phys. Rev. Lett. 83, 3758 (1999); A. J. Bracken, D. Ellinas, and J. G. Wood, J. Phys. A 36, L297 (2003).

[13] M. G. Benedict and A. Czirják, Phys. Rev. A 60, 4034 (1999); P. Foldi, A. Czirjak, B. Molnar, and M. G. Benedict, Opt. Express 10, 376 (2002).

[14] J. Schliemann, J. I. Cirac, M. Kuś, M. Lewenstein, and D.
Loss, Phys. Rev. A 64, 022303 (2001).

[15] R. Paškauskas and L. You, Phys. Rev. A 64, 042310 (2001).

[16] G. C. Ghirardi and L. Marinatto, Phys. Rev. A 70, 012109 (2004).

[17] F. Soto and P. Claverie, J. Math. Phys. 24, 97 (1983).

[18] Our derivation is closely related to the one leading to the Maxwell distribution as the stationary solution of the Boltzmann equation.

[19] J. Botero, M. A. Cirone, J. P. Dahl, A. Delgado, and W. P. Schleich, in The Physics of Communication, Proceedings of XXII Solvay Meeting on the Physics of Information, edited by I. A. V. A. Sadovnichy and H. Walther (World Scientific, Singapore, 2003), p. 568; W. P. Schleich and J. P. Dahl, Phys. Rev. A 65, 052109 (2002).

[20] I. Białynicki-Birula, M. A. Cirone, J. P. Dahl, M. Fedorov, and W. P. Schleich, Phys. Rev. Lett. 89, 060404 (2002); M. A. Andreata and V. V. Dodonov, J. Phys. A 36, 7113 (2003).

[21] A. Ekert and P. L. Knight, Am. J. Phys. 63, 415 (1995).

[22] N. Gisin, Phys. Lett. A 154, 201 (1991).

[23] N. Gisin and A. Peres, Phys. Lett. A 162, 15 (1992).

[24] S. Popescu and D. Rohrlich, Phys. Lett. A 166, 293 (1992).

[25] A. Sommerfeld, Partielle Differentialgleichungen der Physik, 7th ed. (Verlag Harry Deutsch, Thun, Frankfurt/M., 1992).

[26] J. P. Dahl and W. P. Schleich, Phys. Rev. A 65, 022109 (2002).

[27] A more general approach [J. P. Dahl, S. Varro, A. Wolf, and W. P. Schleich (unpublished)] derives an expression for the Wigner function of an $s$ wave starting from the definition, Eq. (37), and performing the angle integrations. 